\title{
The value of genetic testing for inherited retinal disease caused by mutations in the $A B C A 4$ gene in South Africans
}

To the Editor: Gene-based therapies for inherited retinal degenerative diseases (RDDs) have gained momentum in the past 5 years, and there are currently several earlystage clinical trials underway ${ }^{[1]}$ that have the potential to intervene in disease processes and provide clinical benefit to patients. However, knowledge of a patient's specific underlying genetic mutation(s) is a prerequisite for participation in these trials. Identification of the mutations in patients with RDDs is challenging and costly; there are more than 200 genes associated with RDDs, and each can carry hundreds of potential mutations. Finding the one or two mutations responsible for the RDD in a patient is daunting, so the best candidate gene to screen is often determined by the clinical manifestations of the disease and the inheritance pattern observed.

Stargardt disease (STGD) is a macular degeneration that occurs in childhood, resulting in a profound loss of central vision and severely reduced visual acuity. Autosomal recessive STGD is frequently caused by seven specific mutations of the $A B C A 4$ gene in South African whites, particularly those of Afrikaner ancestry. ${ }^{[2]} \mathrm{A}$ 'founder effect' describes when a mutation occurs at a comparatively high frequency in a certain population, because that population has arisen from a small number of ancestors (or founders). Several inherited diseases occur in Afrikaners as a result of founder mutations, e.g. variegate porphyria and familial hypercholesterolaemia. We previously reported that $4.8 \%$ of Afrikaners are unaffected carriers of one of the seven mutations of the $A B C A 4$ gene. ${ }^{[2]}$ This gene has also been implicated in other more severe recessive RDDs, termed ABCA4related retinopathies (AARs). Recessive genetic disorders occur when both the parents are carriers of one mutated allele of the $A B C A 4$ gene, with their other healthy allele compensating, and preventing disease. For the disease to manifest, a child must by chance have inherited two mutated alleles of the $A B C A 4$ gene, one from the father and one from the mother. In order to confirm a genetic diagnosis for the recessive RDD, two mutations in $A B C A 4$ must therefore be identified in the patient with visual impairment.

The aim of a recent study was to determine whether a locally developed test for the seven common mutations, 
called 'Quick 7' (which provides a genetic diagnosis, i.e. identification of both mutations, in $40 \%$ of Afrikaner patients with STGD ${ }^{[2]}$ ), could be effective in screening South Africans with other AARs, such as cone-rod dystrophy (CRD), retinitis pigmentosa (RP), and fundus flavimaculatis (FFM). Twenty patients with CRD, 4 with FFM and 40 with RP were screened, and although the test provided a diagnosis for $25 \%$ of patients with FFM and $10 \%$ of those with CRD, it was ineffectual in the diagnosis of RP. The vast majority of the patients in this cohort were white (79.7\%). Although the number of patients screened is small, the trend observed supports a previously proposed model ${ }^{[3]}$ whereby the 'severity' of the gene defect is proportional to the severity of the disease: if the combination of the two mutations is 'severe', the disease will manifest more severely, e.g. RP; if the combination of the two mutations in the gene is milder, the disease manifestation will be milder, e.g. STGD or FFM. Our finding that 25\% of CRD patients carry only one of the seven mutations indicates that their (as yet unfound) second mutation is more severe. Furthermore, only one RP patient had a partial result, suggesting that for this AAR, both mutations are severe. In summary, it appears that the seven common mutations are more likely to cause relatively mild disorders such as STGD and the clinically similar FFM. Different, more severe mutations are probably involved in CRD and RP. Therefore, while this 'Quick 7' assay (requested via the National Health Laboratory Service) is valuable and effective for the diagnosis of STGD, it should not be offered for other AARs, which are likely to be caused by a different spectrum of mutations.

The clinical manifestations of RDD may overlap with each other or change with disease progression. Patients may therefore find themselves being given different clinical diagnoses and prognoses over time, which may be confusing and frustrating, whereas the genetic diagnosis remains unequivocal. A confirmed genetic diagnosis also allows testing of the extended family, providing clearer risks for individuals and enabling life planning and decisions on reproductive options. For those who do not carry the family mutations, the fear of potentially developing the familial visual impairment can be eliminated. For those affected by the disease associated with the $A B C A 4$ mutations, there is additional information that can be provided to assist with clinical management. Animal studies have shown that high doses of vitamin A (which is sometimes prescribed indiscriminately for patients with RDDs) and light exposure may exacerbate vision loss when the ABCA4 protein is not functioning properly. ${ }^{[4,5]}$ These patients can be cautioned to avoid excessive vitamin A supplementation and to wear appropriate protective sunglasses in bright light, in order to preserve their vision. This advice does not necessarily apply to other RDDs with different underlying genetic defects. Finally, to date our research has resulted in the genetic diagnosis of more than 160 families with $A B C A 4$ mutations, who are now eligible to participate in or benefit from StarGen treatment, the $A B C A 4$-gene replacement therapy trials currently in progress. ${ }^{[6]}$

\section{J Roberts}

S Hardie

T Goolam Hoosen

R S Ramesar

\section{J Greenberg}

UCT/MRC Human Genetics Research Unit, Division of Human Genetics, Department of Clinical Laboratory Sciences, Institute of Infectious Disease and Molecular Medicine, Faculty of Health Sciences, University of Cape Town, South Africa lisa.roberts@uct.ac.za

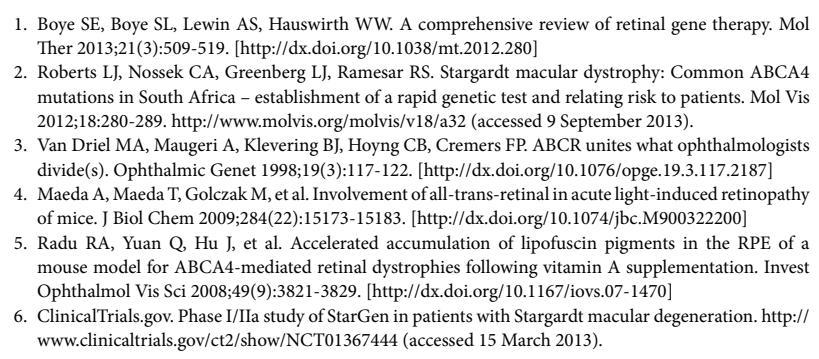
Ther 2013;21(3):509-519. [http://dx.doi.org/10.1038/mt.2012.280]

2. Roberts LJ, Nossek CA, Greenberg LJ, Ramesar RS. Stargardt macular dystrophy: Common ABCA4 mutations in South Africa - establishment of a rapid genetic test and relating risk to patients. Mol Vis 2012;18:280-289. http://www.molvis.org/molvis/v18/a32 (accessed 9 September 2013).

3. Van Driel MA, Maugeri A, Klevering BJ, Hoyng CB, Cremers FP. ABCR unites what ophthalmologists divide(s). Ophthalmic Genet 1998;19(3):117-122. [http://dx.doi.org/10.1076/opge.19.3.117.2187]

4. Maeda A, Maeda T, Golczak M, et al. Involvement of all-trans-retinal in acute light-induced retinopathy of mice. J Biol Chem 2009;284(22):15173-15183. [http://dx.doi.org/10.1074/jbc.M900322200]

5. Radu RA, Yuan Q, Hu J, et al. Accelerated accumulation of lipofuscin pigments in the RPE of a mouse model for ABCA4-mediated retinal dystrophies following vitamin A supplementation. Invest
mosition mouse model for ABCA4-mediated retinal dystrophies following vitamin A supplen

6. ClinicalTrials.gov. Phase I/IIa study of StarGen in patients with Stargardt macular degeneration. http:// www.clinicaltrials.gov/ct2/show/NCT01367444 (accessed 15 March 2013).

S Afr Med J 2013;103(10):702-703. DOI:10.7196/SAMJ.6948 